\title{
Design and Measurement Data for a Microwave Dual-CP Antenna Using a New Traveling-Wave Feed Concept
}

\author{
Kum Meng Lum, Timo Tick, Charles Free, Member, IEEE, and Heli Jantunen
}

\begin{abstract}
Design and measurement data are presented that show how the novel concept of a traveling-wave-fed circular-polarization (CP) microstrip antenna can be extended to provide an antenna with dual CP. A new refinement to the basic antenna structure is also introduced that shows that the efficiency of radiation can be enhanced by using a dual dielectric to improve the fringing fields at the edges of the radiating patches by around 40\%. Furthermore, new theoretical and simulated data are introduced to support the original traveling-wave feed concept.
\end{abstract}

Index Terms-Circular polarization (CP), dual polarization, patch antenna, traveling wave.

\section{INTRODUCTION}

A NEW concept, whereby a traveling-wave system was used to feed a circular-polarized (CP) microstrip patch array, was established by Lum et al. in [1] and [2]. Lum et al. presented practical data from 5-GHz prototype antennas to support the new concept. In this paper, we have introduced a significant extension to the basic design by using two parallel slotline feeds to produce a dual-polarization antenna. Practical data are presented that verify the principle of the new dual-polarization antenna.

The basis of this study, as reported in the European Microwave Conference [2], was originally established by Huang [3], who used four probe-fed patches and a corporate feed structure. In our case, the probe feeds are replaced with a circular slotline channel of width $W_{s}$, which runs in the ground plane beneath the patches. The concept of using a slotline to excite a single patch was originally reported by Tang et al. [4]. A traveling wave was excited in the slotline channel through a microstrip-to-slotline transition [5], [6].

One of the benefits of the antenna structure under investigation is that the antenna is particularly suitable for inclusion in highly integrated multilayer packages. Related to this, two additional features have been investigated, namely, the use of a low-temperature co-fired ceramic (LTCC) structure and the use of mixed dielectrics. It is well known that LTCC offers a number of advantages for the fabrication of microwave circuits. Data is

Manuscript received October 7, 2005; revised January 5, 2006.

K. M. Lum is with the Institute of Infocomm Research, Singapore 550211 (e-mail: maxlum78@hotmail.com).

T. Tick and H. Jantunen are with the Microelectronics and Materials Physics Laboratories, Department of Electrical and Information Engineering, University of Oulu, Oulu 90014, Finland.

C. Free is with the School of Electronics and Physical Sciences, University of Surrey, Surrey GU2 7XH, U.K.

Digital Object Identifier 10.1109/TMTT.2006.875463

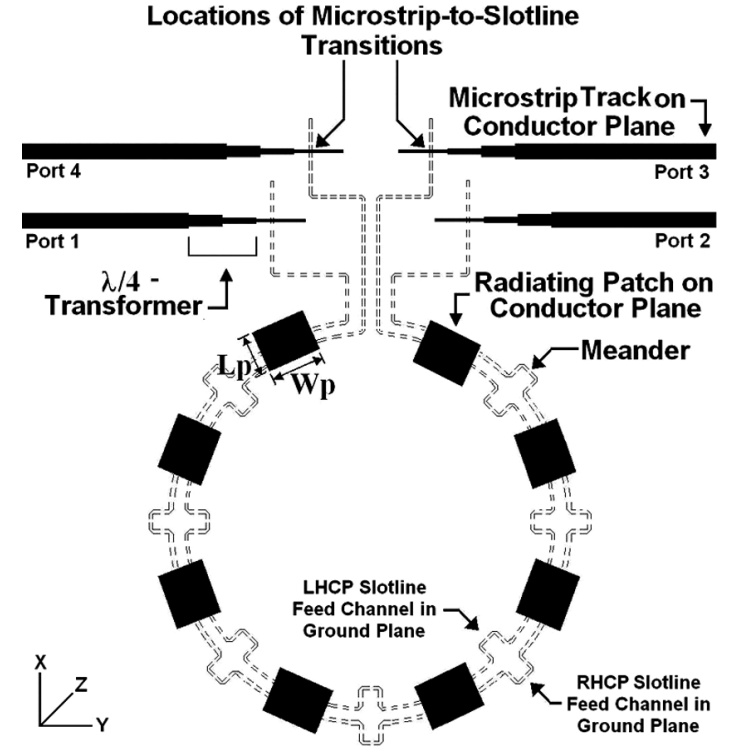

Fig. 1. Dual-CP antenna configuration.

presented on the practical performance of a $15-\mathrm{GHz} \mathrm{CP}$ antenna based on the new traveling-wave feed principle. Multilayer fabrication techniques, using thick-film or LTCC technology, also offer the potential for having layers of different permittivity. It is shown that this feature can be used to advantage by incorporating a low dielectric layer beneath the patches so as to increase the magnitude of the fringing field and, hence, increase the magnitude of the radiated signal.

\section{Dual-CP Antenna Structure}

The structure of the dual-polarization CP antenna is depicted in Fig. 1. It can be seen that the microstrip patches are fed through two parallel slots in the ground plane. A signal applied at port 1 will excite a signal traveling in the counterclockwise direction in the outer of the two slots, and this will produce right-handed circular polarization (RHCP). Similarly, a signal applied at port 3 will excite a signal traveling clockwise in the inner slot, and this will excite the left-handed circular polarized (LHCP) signal. It can be seen that this is an extension of the single-slot antenna reported by the authors as in [2]. The key aspect of this structure is that there should be a distance of $\lambda / 8$ between the feed points to the patches along the slotline. In order to reduce the overall size of the structure, a meander was inserted into the slotline sections between each patch so as to reduce the 


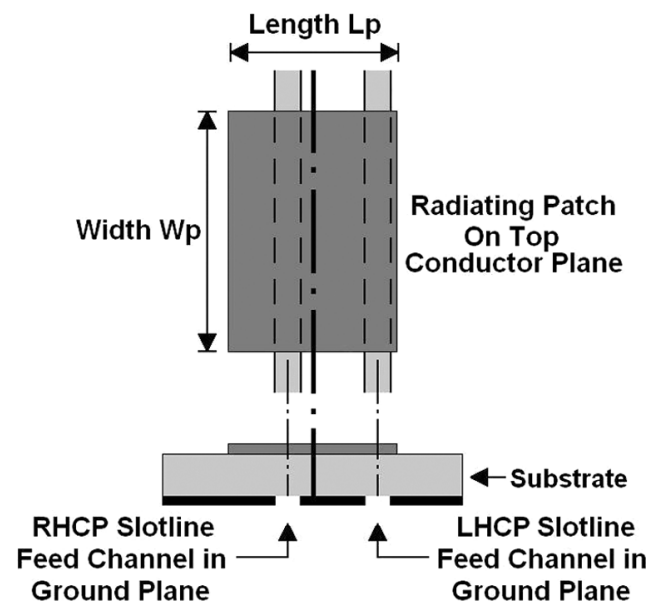

Fig. 2. Dual-CP antenna slotline arrangement.

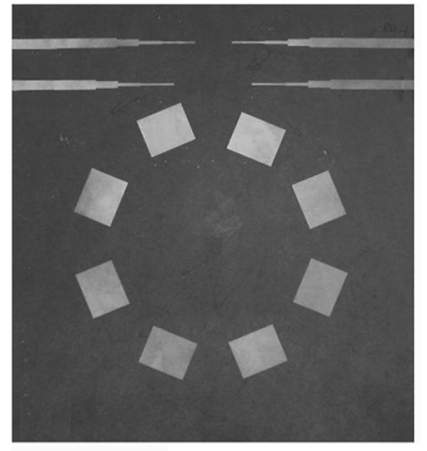

(a)

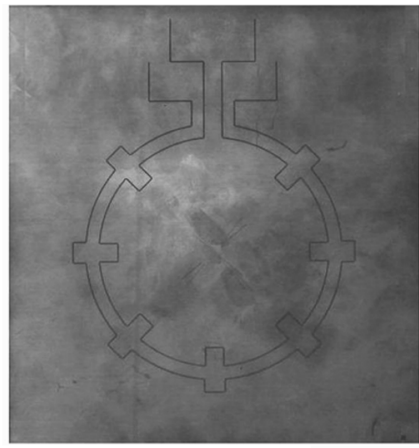

(b)
Fig. 3. Fabricated dual-CP Antenna. (a) Top plane. (b) Bottom plane.

mean diameter. It should be noted that, for both of the slot excitations, there is a progressive offset of the patches relative to the slotline, as the signal propagates around the appropriate slot. This incremental offset is to ensure that each patch will receive approximately the same amount of coupled energy as the signal propagates around the slot, gradually decreasing in amplitude. The offset of the slots relative to the patches is shown in Fig. 2. In order to prevent coupling between the slots, the spacing between the slots was maintained at $5 w$, where $w$ is the width of the slot. It was found, both through simulation and experiment, that this spacing maintained the coupling between the slots below $25 \mathrm{~dB}$. It should be noted that the slotline channel is relatively narrow so that little signal was radiated from the feed.

Photographs of the 5-GHz test antennas are shown in Fig. 3. These test antennas were fabricated on RT/Duroid 5870 in which the substrate had a relative permittivity of 2.33 and a thickness of $1 \mathrm{~mm}$. It should be noted that since only two conductor layers were used in the test antennas, it was necessary to include the microstrip feed lines on the same surface as the radiating patches. This would be a problem if the antennas were used for receiving, as signals could be picked up directly on the microstrip feed lines. However, the problem is resolved if a three-conductor structure is used. This is demonstrated later in this paper when a three-conductor layer LTCC antenna is described.

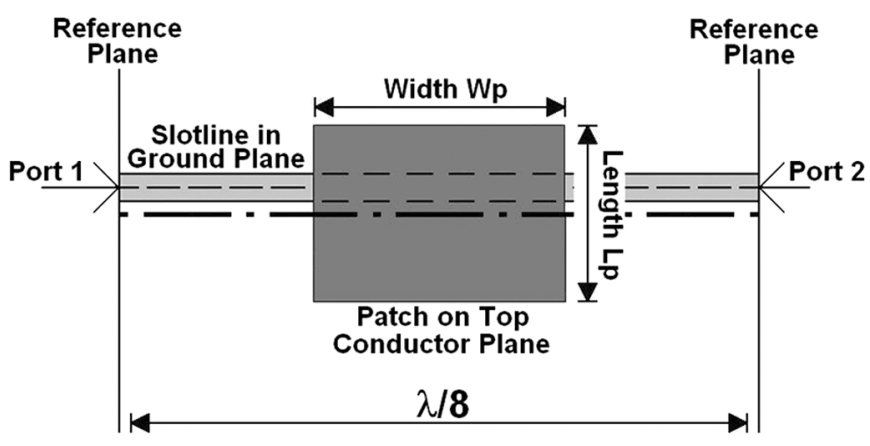

Fig. 4. Two-port network representation of a single patch.

The key dimensional data for the antennas shown in Fig. 3 are $W_{p}=23.000 \mathrm{~mm}, L_{p}=19.000 \mathrm{~mm}$, incremental offset $=$ $700 \mu \mathrm{m}$, and slotline width $W_{s}=500 \mu \mathrm{m}$.

\section{ANTENNA THEORY}

A theoretical analysis using matrix multiplication was used to study the behavior of a single-slot-fed antenna array of the type described in [2], which incorporated a 500- $\mu$ m incremental offset. Initially, each patch and its associated slotline feed was represented by a two-port network in terms of scattering parameters. The reference planes for each slot-fed patch are shown in Fig. 4. It should be noted that a spacing of $\lambda / 8$ between the reference planes was chosen so that successive patches could be added without the need for adding additional sections of slotline.

The scattering parameters of each successive patch, with the incremental offset of $500 \mu \mathrm{m}$, were obtained through simulation using Momentum. After obtaining the $A B C D$ matrix for the individual networks, the overall $A B C D$ representation was obtained through matrix multiplication. The validity of the analysis was demonstrated when the overall $A B C D$ representations were converted back to $S$-parameters and compared with measurement. For example, the theoretical $S_{11}$ value of $-11.5 \mathrm{~dB}$ compared very well with the measured value of $-12.3 \mathrm{~dB}$, which was presented in [2].

To aid the understanding of the behavior of individual patches, plots are given in Figs. 5 and 6 that show how $S_{11}$ and $S_{21}$ for an individual patch vary with the incremental offset. In Fig. 5, we see that there is a greater change in $S_{11}$ for small and large values of incremental offset. This can be explained in terms of conventional transmission-line theory and the standing wave that will exist in the patch at resonance. Fig. 6 shows a well-behaved characteristic, in which $S_{21}$ decreases steadily with an increase in the offset, which is reasonable since more power will be lost through the patch due to radiation as the offset increases. It can be shown that $S_{21}$ as a function of incremental offset gives a logarithmic characteristic. The usefulness of this study is that we can predict the effect of the offset and, thus, design future arrays without the need to simulate the effects of all the individual patches. Through correct design of the offsets, it can also be arranged that the majority of the energy traveling in the slotline will be coupled onto the radiating patches with minimum energy dissipated in the slotline termination. 


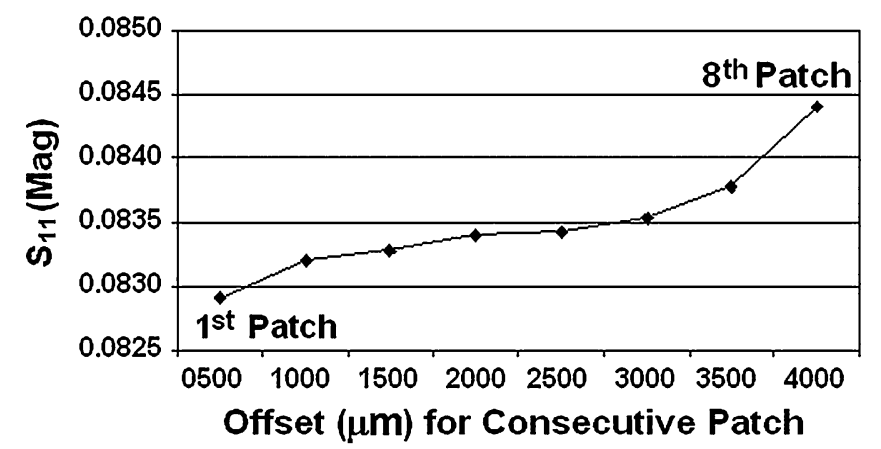

Fig. 5. Plot of $S_{11}$ magnitude against amount of offset.

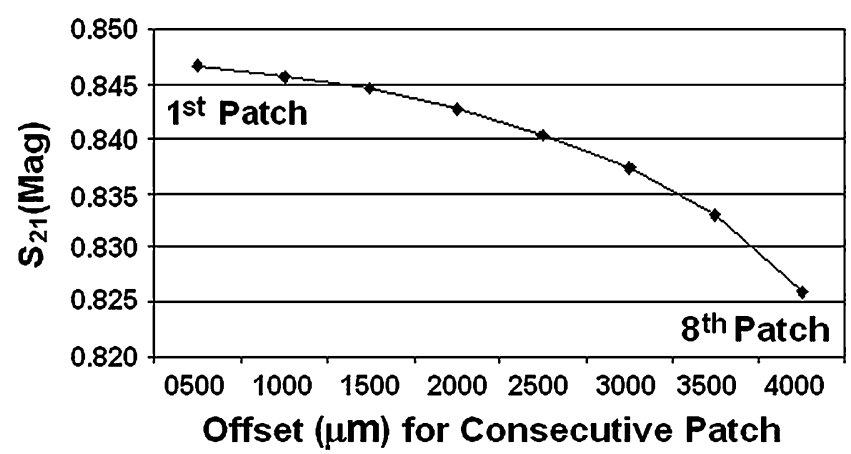

Fig. 6. Plot of $S_{21}$ magnitude against amount of offset.

TABLE I

SUMMARY OF OPTIMUM MEASURED RESULTS FOR CP ANTENNA WITH 700- $\mu$ m INCREMENTAL OFFSET AT $5.04 \mathrm{GHz}$

\begin{tabular}{ll}
\hline \hline \multicolumn{1}{c}{ Parameter } & Values \\
\hline Return loss & $-27.6 \mathrm{~dB}$ \\
Return loss bandwidth & $8.2 \%$ \\
Front to back ratio & $20.7 \mathrm{~dB}$ \\
Efficiency & $95 \%$ \\
Axial ratio & $0.7 \mathrm{~dB}$ \\
Cross polarization & $-21.2 \mathrm{~dB}$ \\
\hline \hline
\end{tabular}

\section{Summary of Key Results for SINGLE-SLOT CP ANTENNA}

In order to make a proper comparison of the performance of the dual-slot antenna, we have summarized the key parameters of a single-slot antenna, already published in [2]. Table I summarizes the optimum measured results for a single-slot antenna with $700-\mu \mathrm{m}$ incremental offset at $5.04 \mathrm{GHz}$. Fig. 7 shows the high quality of circular polarization that can be obtained using this new technique with a single-slot feed.

The results for the eight-patch array show a significant improvement in gain when compared with the results for the fourpatch array reported in [1].

\section{Dual-CP Antenna Measurement Results}

As shown in Fig. 8, the best match for the dual-polarization antenna was obtained at $4.99 \mathrm{GHz}$ with an $S_{11}$ value less than $-30 \mathrm{~dB}$. This matching frequency is close to the designed value of $5 \mathrm{GHz}$. The bandwidth of the proposed antenna (VSWR $<2$ ) is approximately $21.2 \%$. The maximum gain measured over this bandwidth is approximately $14.8 \mathrm{dBi}$. Fig. 9 shows the isolation between the two slotlines. The best isolation is obtained close

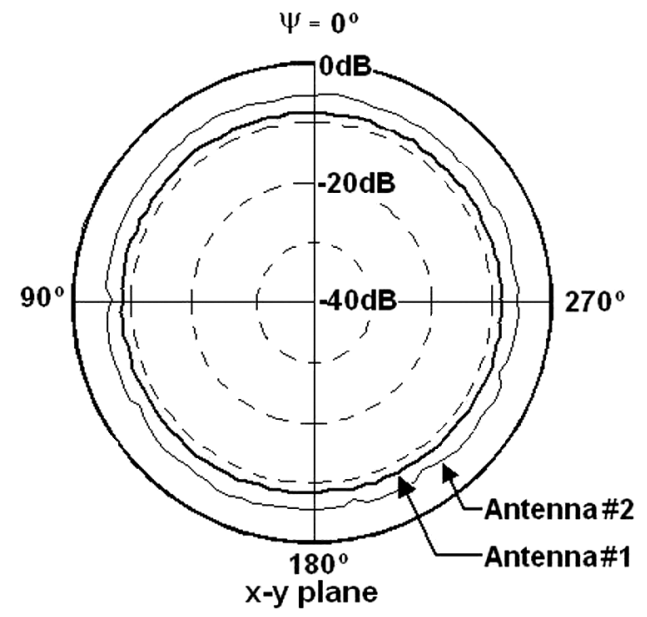

Antenna \#1 $500(\mu \mathrm{m})$ incremental offset Antenna \#2 $700(\mu \mathrm{m})$ incremental offset -

Fig. 7. Measured CP for single-slot feed antenna with 500- and 700- $\mu \mathrm{m}$ incremental offset.

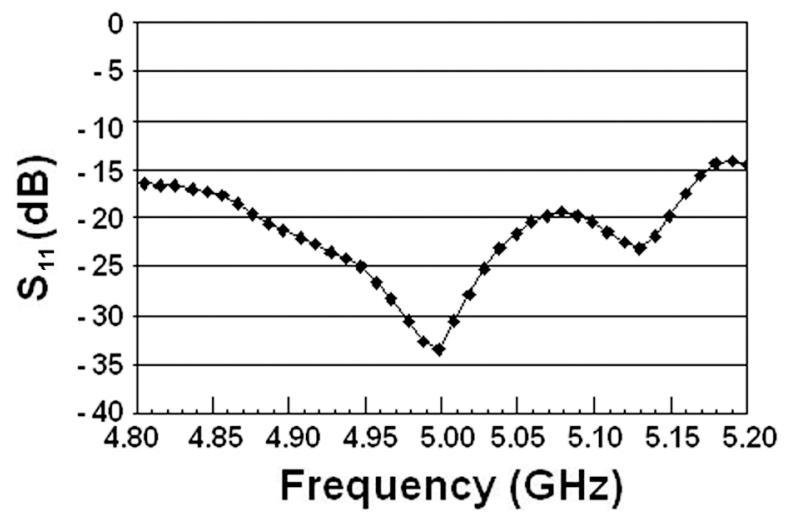

Fig. 8. Measured $S_{11}$ for dual-CP antenna.

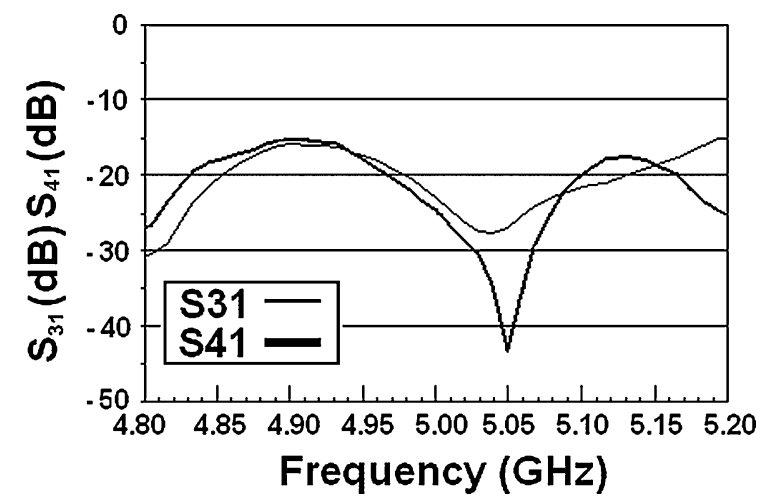

Fig. 9. Measured $S_{31}$ and $S_{41}$ for dual-CP antenna.

to the designed frequency of $5 \mathrm{GHz}$, with an $S_{31}$ value less than $-25 \mathrm{~dB}$ at $5.02 \mathrm{GHz}$ and $S_{41}$ value less than $-40 \mathrm{~dB}$ at $5.05 \mathrm{GHz}$. (the port identification is given in Fig. 1).

Figs. 10 and 11 show a comparison of the simulated and measured data for the 3-dB axial ratio of the LHCP and RHCP. The optimum measured 3-dB axial ratio of the LHCP and RHCP are $2.7 \mathrm{~dB}$ at $4.95 \mathrm{GHz}$ and $2.1 \mathrm{~dB}$ at $5.05 \mathrm{GHz}$ with $3-\mathrm{dB}$ axial ratio bandwidth of $7 \%$ and $11 \%$, respectively. The corresponding simulated and measured polarization quality of the LHCP and RHCP 


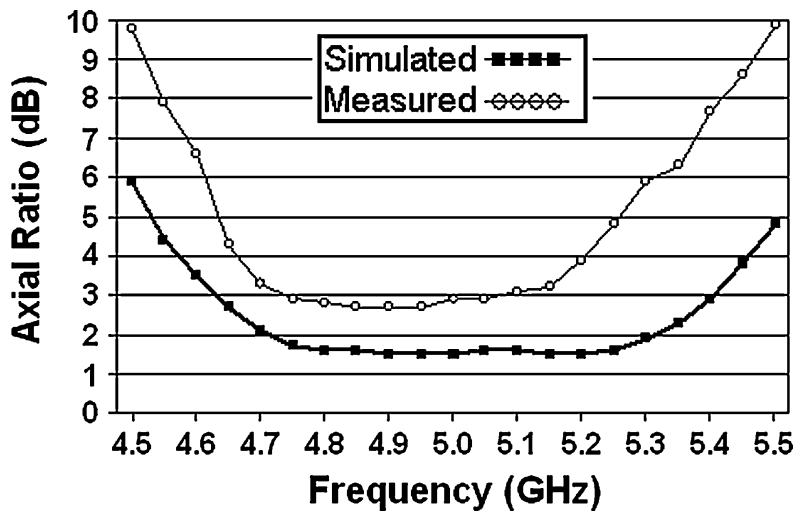

Fig. 10. Simulated and measured 3-dB axial ratio for LHCP.

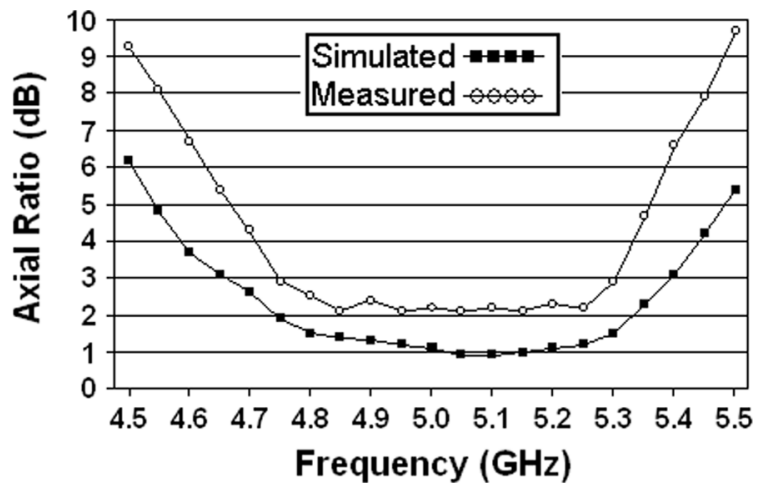

Fig. 11. Simulated and measured 3-dB axial ratio for RHCP.

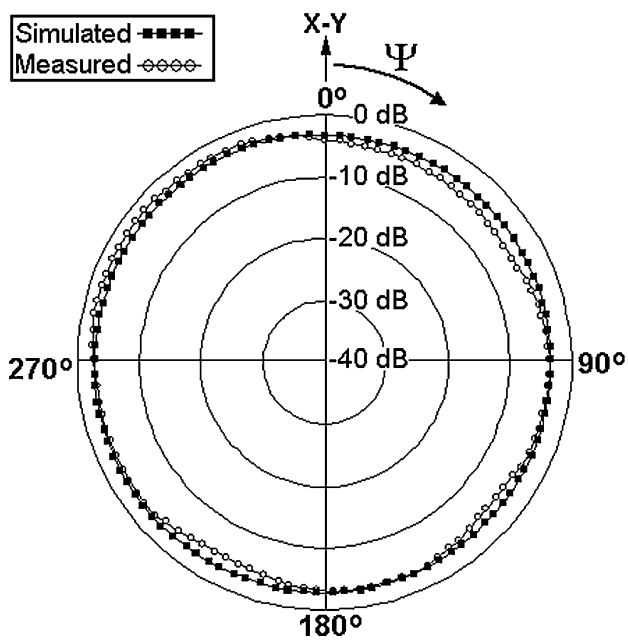

Fig. 12. Simulated and measured circular polarization for LHCP.

are represented in Figs. 12 and 13, which shows the relative magnitudes of the LHCP and RHCP as a function of spatial angle. It can be seen that the high quality of circular polarization obtained with a single slot, and displayed in Fig. 7, has been maintained.

Some reductions in the $\mathrm{CP}$ quality may be due to received signals being picked up directly on the microstrip lines during the measurement process. This problem can be solved by implementing the multilayer structure, as introduced in Section VI, to allow the microstrip feed line to be on the reverse side of the antenna.

The simulated and measured radiation patterns for both hands of polarization are shown in Figs. 14 and 15, and it can be seen that essentially the same characteristics are obtained in each case.

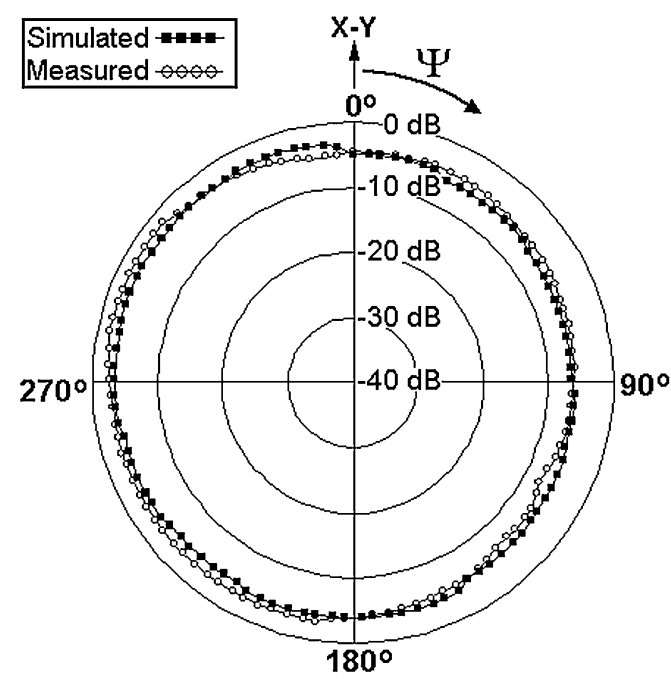

Fig. 13. Simulated and measured circular polarization for RHCP.

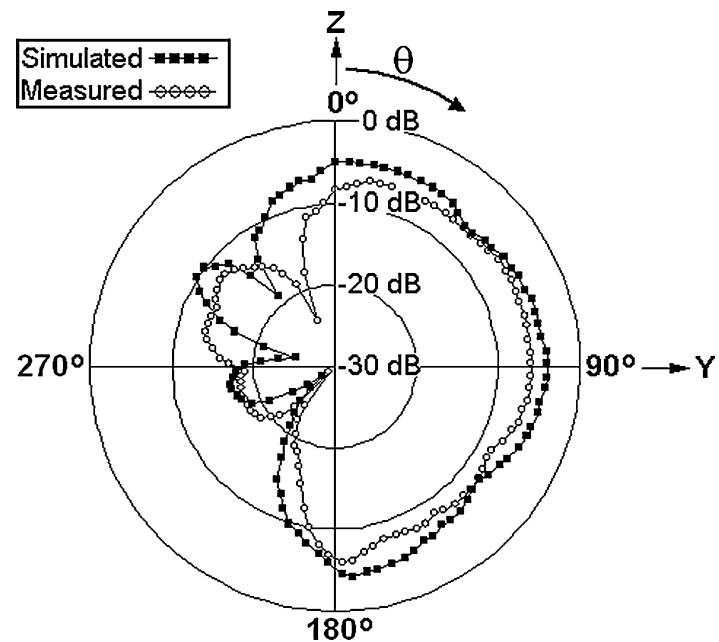

Fig. 14. Simulated and measured radiation pattern for LHCP.

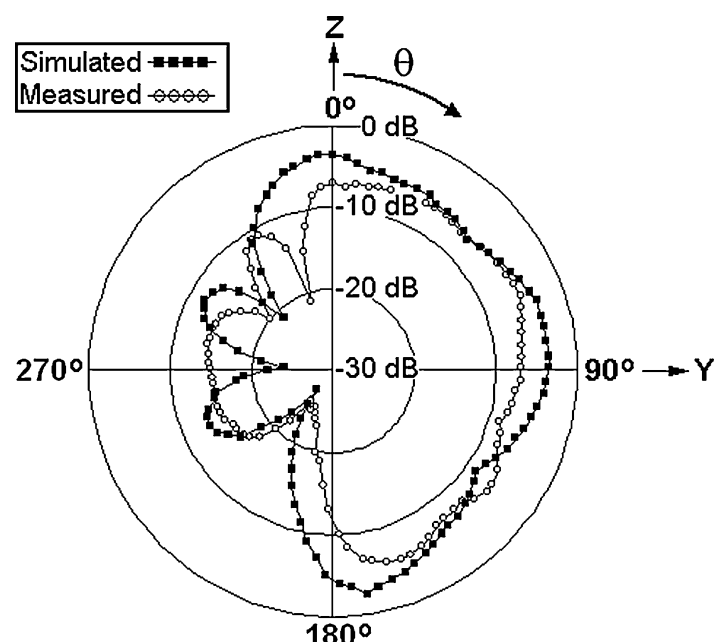

Fig. 15. Simulated and measured radiation pattern for RHCP.

\section{Multilayer LTCC CP Antenna Structure AND MEASUREMENTS}

In order to validate the new design, a single-slot-fed antenna was fabricated and tested using a different material (LTCC) and at a different frequency $(15 \mathrm{GHz})$. The structure of the antenna 


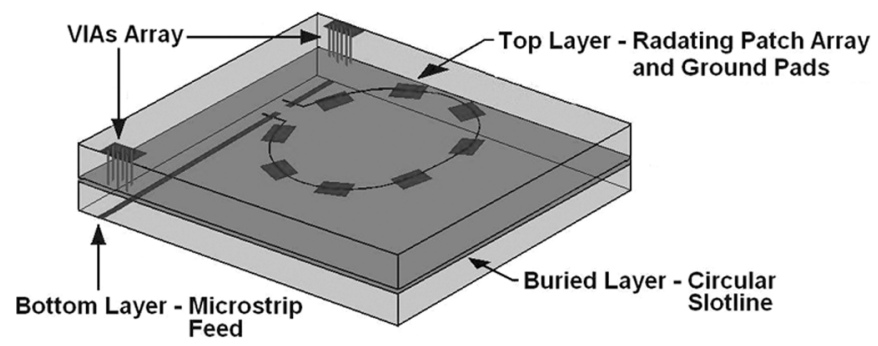

Fig. 16. Multilayer LTCC antenna configuration.

\section{Conductor Layers}

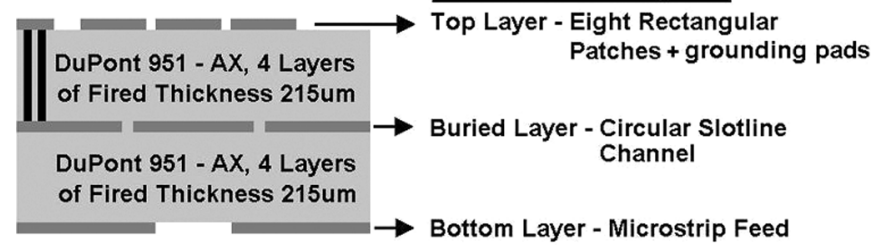

Fig. 17. Multilayer LTCC antenna layer configuration.

is shown in Figs. 16 and 17. The multilayered nature of the structure permitted the microstrip feed lines to be positioned on the opposite side to the radiating patches, thus enabling the antenna to be used for receiving. The LTCC antennas consisted of two thick layers of dielectric, each composed of four layers of DuPont 951 LTCC tape (245- $\mu$ m thick). The slotline feeding the patches was formed in a buried ground. Vias were used to make an electrical connection between pads on the upper surface and the buried ground plane. The pads were necessary to earth the subminiature A (SMA) connectors. The vias were laser drilled and filled with an injection via filler using DuPont $6141 \mathrm{Ag}$ via paste. The diameter of the vias was $200 \mu \mathrm{m}$. The buried ground plane and the patches on the top surface were screen printed using DuPont $6142 \mathrm{Ag}$ conductor paste and co-fired. Finally, the feed lines on the bottom side were printed using DuPont 6160 silver paste, and after post firing, the antennas were cut from the panel to their final size.

The large uniform ground plane in this structure, approximately $65 \mathrm{~mm} \times 65 \mathrm{~mm}$, caused extra challenges for manufacturing. The main danger was substrate warping caused by the shrinkage mismatch of the conductive paste and the ceramic sheet. However, in this case, the ground plane was located symmetrically in the center of the substrate and, thus, the shrinkage of the plane is equally restricted from both sides and warping was avoided. The burnout period of the firing profile given by DuPont was also revised. To ensure the removal of all organics, a very slow heating rate of $2{ }^{\circ} \mathrm{C} / \mathrm{min}$ from room temperature up to $500^{\circ} \mathrm{C}$, and $1-\mathrm{h}$ dwell time at this temperature was used. This process provided a defect-free structure without any interlayer delamination and surface blistering, which are likely to occur with this kind of high-volume substrate having large uniform ground planes.

The antenna that was fabricated on LTCC DuPont 951 material had the following parameters:

1) single fired thickness: $h=245 \mu \mathrm{m}$;

2) dielectric constant: $\varepsilon_{r}=7.8$;

3) loss tangent: $\tan \delta=0.0015$.

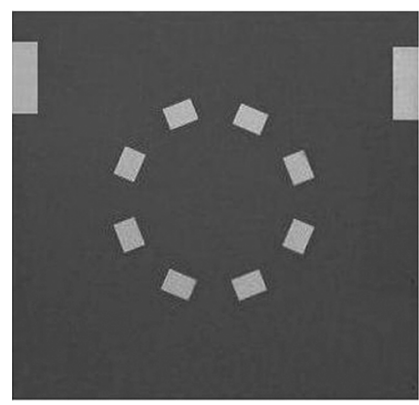

(a)

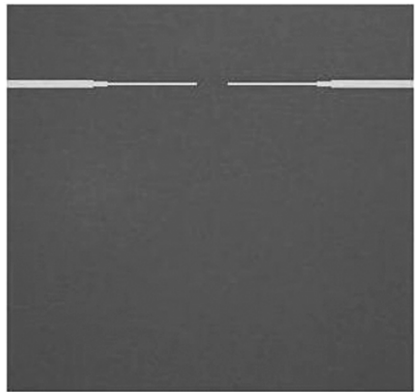

(b)
Fig. 18. Fabricated multilayer LTCC antenna. (a) Top plane. (b). Bottom plane.

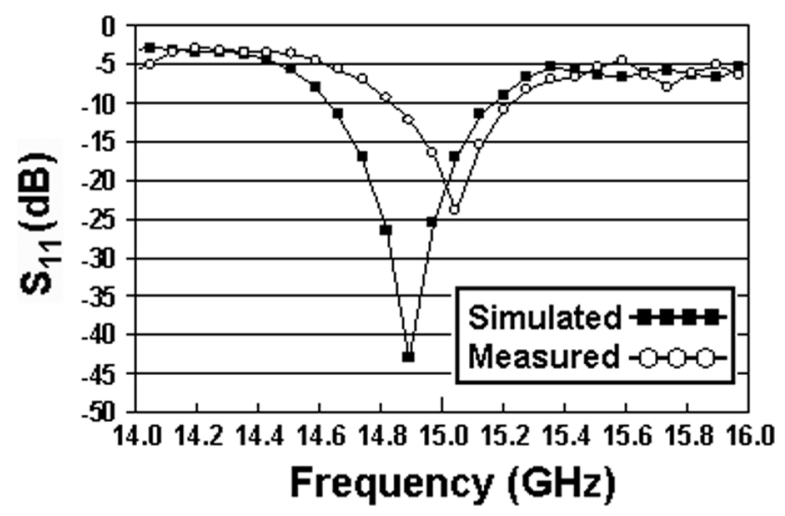

Fig. 19. Simulated and measured $S_{11}$ for multilayer LTCC antenna.

The incremental offset of the patches was set at $100 \mu \mathrm{m}$ with a slotline width $W_{s}, 200 \mu \mathrm{m}$. The patch dimensions were $L_{p}=$ $3580 \mu \mathrm{m}$ and $W_{p}=4770 \mu \mathrm{m}$. As with the other antennas that were investigated, the patches were spaced $\lambda / 8$ along the circular slotline.

Photographs of the fabricated LTCC antenna array are shown in Fig. 18(a) and (b), where (a) is the top view and (b) is the bottom view of the substrate. View for the slotline channel is not feasible since it is located in the buried ground plane. Overall circuit dimension is $65 \mathrm{~mm} \times 65 \mathrm{~mm}$.

Comparison of the simulated and measured data for the return loss are presented in Fig. 19. Optimum measured return loss was obtained at $15.04 \mathrm{GHz}$ with values less than $-20 \mathrm{~dB}$. Bandwidth of the LTCC antenna array (voltage standing-wave ratio $($ VSWR) $<2$ ) is $2.7 \%$ and the corresponding optimum measured gain over this bandwidth is approximately $7.8 \mathrm{dBi}$. Reasonably good agreement between the simulated and measured radiation pattern of the antenna is demonstrated at $15.03 \mathrm{GHz}$, as shown in Fig. 20.

Good quality circular polarization existed at $14.90 \mathrm{GHz}$, as shown in Fig. 21. This is verified by the simulated and measured data of the 3-dB axial ratio, as plotted in Fig. 22. The 3-dB axial ratio bandwidth of the LTCC antenna array is $1.7 \%$.

\section{Multilayer Thick-Film Radiation ENHANCED PATCH ANTENNA STRUCTURE AND THEORY}

It is well known that there will be a concentration of charge along the edges of a resonant patch, giving rise to fringing fields. 


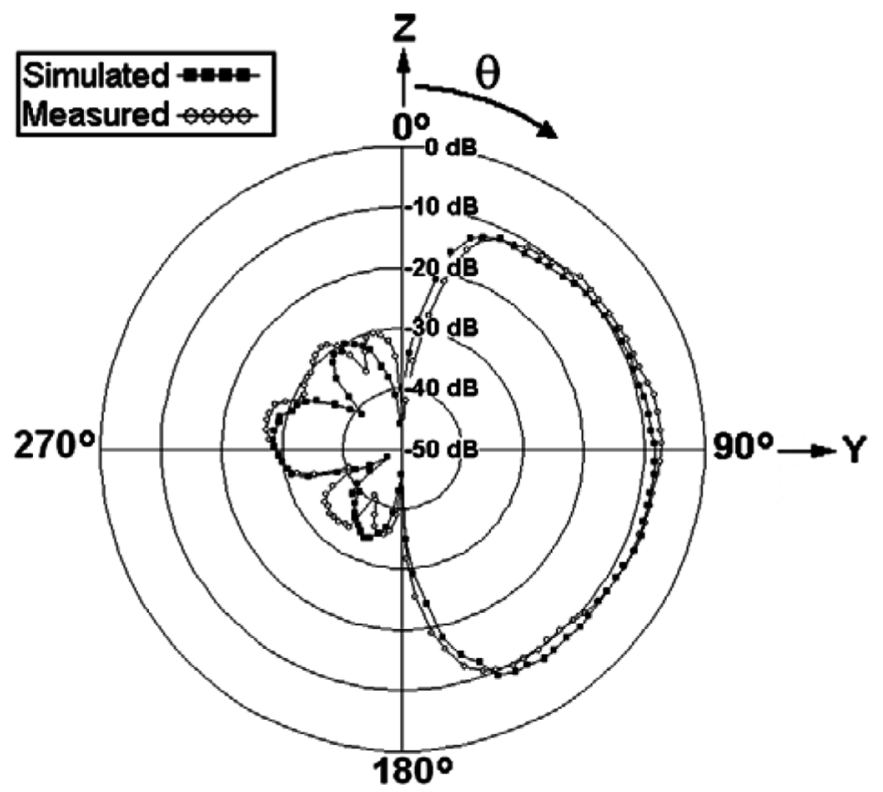

Fig. 20. Simulated and measured radiation pattern for multilayer LTCC antenna.

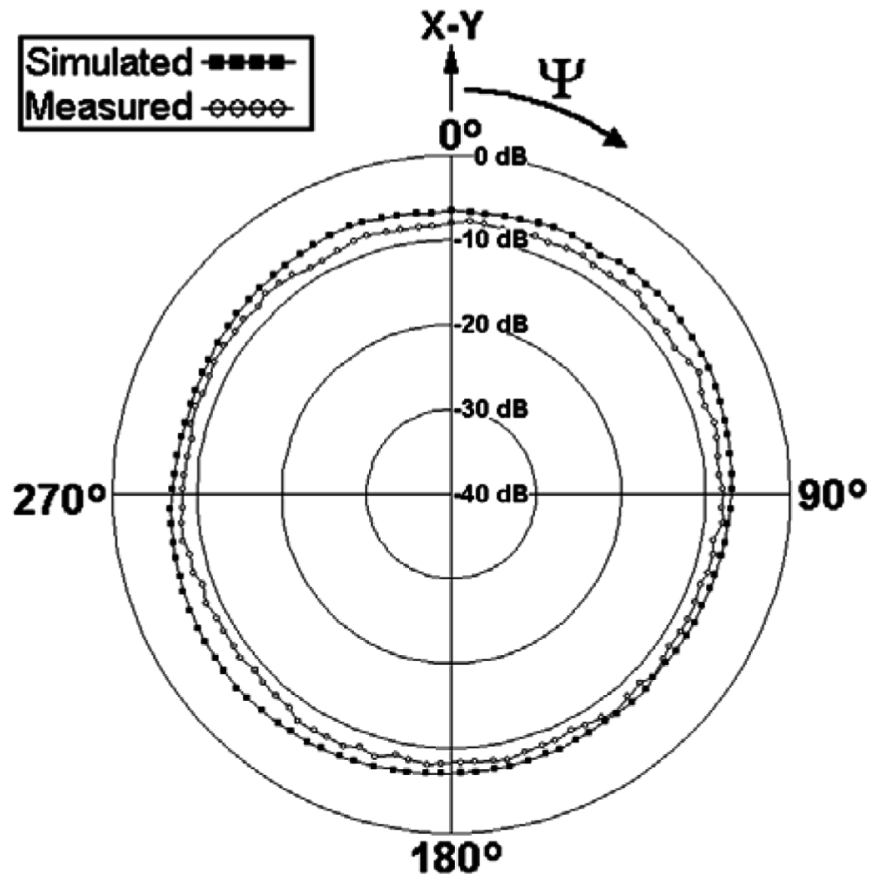

Fig. 21. Simulated and measured circular polarization for multilayer LTCC antenna.

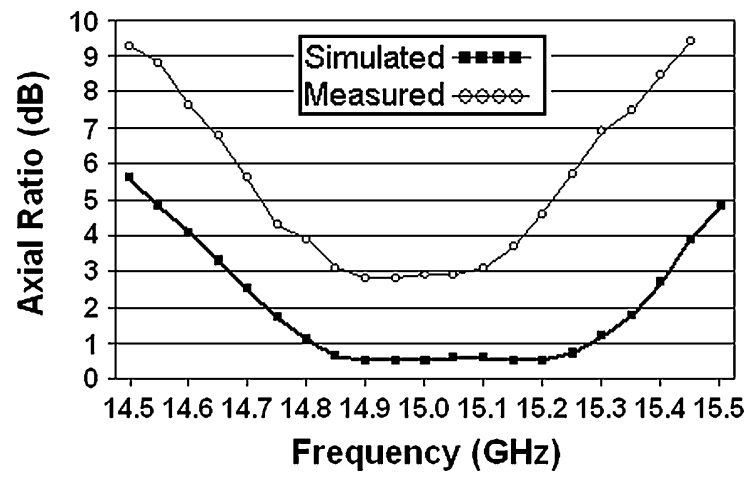

Fig. 22. Simulated and measured 3-dB axial ratio for multilayer LTCC antenna.

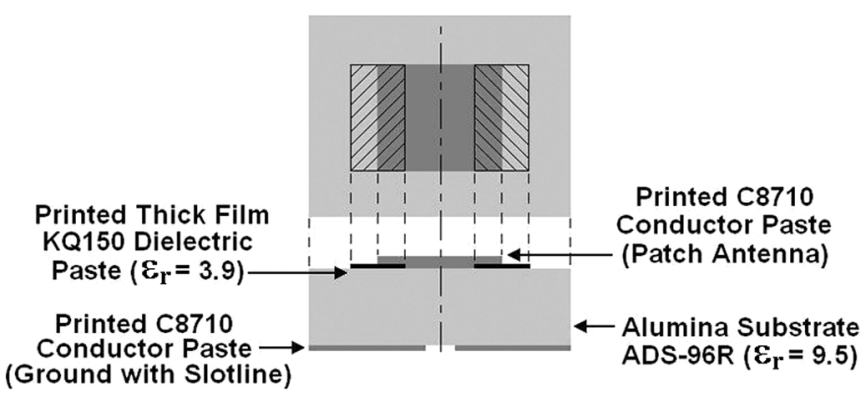

Fig. 23. Dual low dielectric-constant regions configuration.

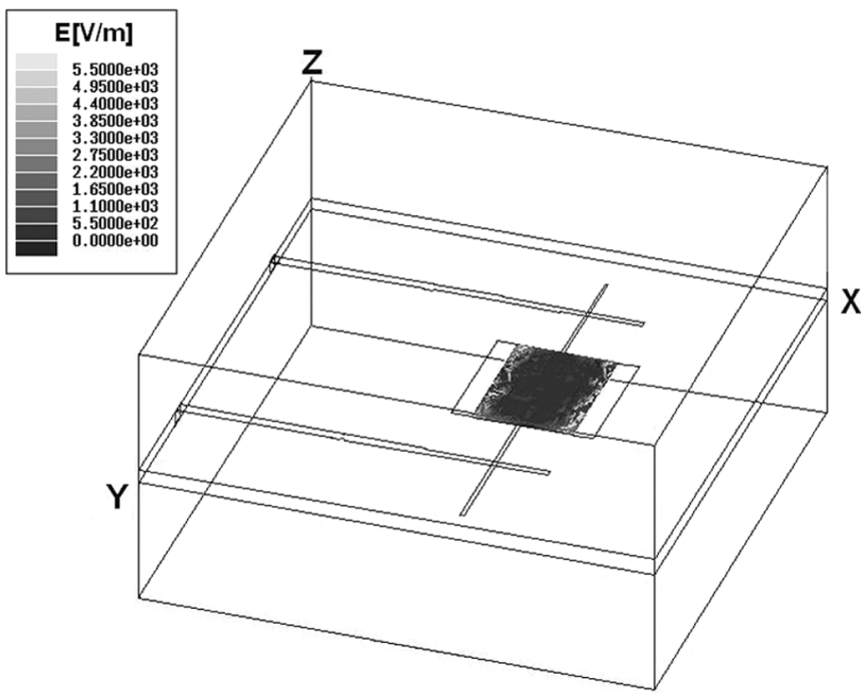

Fig. 24. HFSS modeling of dual low dielectric-constant regions configuration incorporated with $1000-\mu \mathrm{m}$ offset between center of the slotline and center of the patch.

In order to improve the efficiency of radiation of the new structure, a theoretical investigation based on electromagnetic simulation was performed to examine the effect of placing strips of low dielectric-constant material beneath the radiating edges of the patch to enhance the fringing fields

As shown in Fig. 23, two thin regions of low dielectric-constant thick-film dielectric material is arranged beneath and in parallel with the radiating edges of the patch. Due to the presence of the low dielectric-constant material, repulsive forces of the like charges on the bottom surface of the patch are strengthened. Thus, the fringing fields at the radiating edges significantly improved, while the effect on the overall patch dimensions is negligible.

Fig. 24 shows the model used in HFSS; this shows the two regions of low dielectric constant, with the slotline offset by $1000 \mu \mathrm{m}$ from the center of the patch. The same basic model, but without the low dielectric regions, was then used to verify the enhanced performance due to the inclusion of these regions.

It can be seen from Fig. 25(a) and (b), that the presence of the low dielectric material increases the intensity of the electric field distribution at the patch edges by around $40 \%$. To further establish the validity of this unique technique, a greater offset of $2000 \mu \mathrm{m}$ was considered, and the results are shown in Fig. 25(c) and (d). Again we see an increase in the radiation intensity of around $40 \%$. 


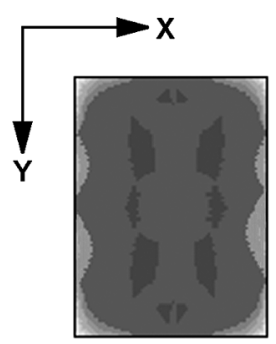

(a)

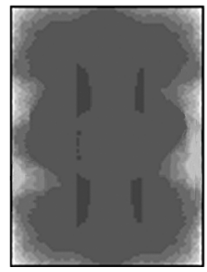

(c)

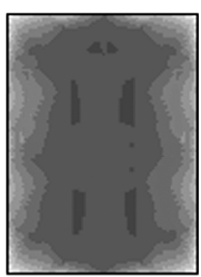

(b)

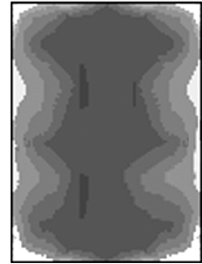

(d)
Fig. 25. HFSS top-view modeling of electric field distribution of patch antenna. (a) $1000-\mu \mathrm{m}$ offset. (b) $1000-\mu \mathrm{m}$ offset with two regions of low dielectric constant material. (c) $2000-\mu \mathrm{m}$ offset. (d) $2000-\mu \mathrm{m}$ offset with two regions of low dielectric-constant material.

Thus the concept of using low dielectric-constant material sections has been verified from the results of simulation. The technique seems particularly suitable for use with the $\mathrm{CP}$ antennas under consideration because of the general suitability of the whole structure for implementation in multilayer thick-film or LTCC formats.

\section{CONCLUSION}

The concept of a new dual-polarization CP antenna has been established through simulation and practical measurement. Moreover, it has been shown that the technique can be successfully employed at different frequencies, namely, 5 and $15 \mathrm{GHz}$. Two different processes were used to manufacture test antennas, conventional etching, and LTCC and, in both cases, the results were very good. A radiation enhancement method, using regions of low dielectric constant, has been demonstrated both through simulation and measurement. The technique seems particularly suitable for use in multilayer microwave circuits, and particularly highly integrated multilayer transceiver packages.

\section{ACKNOWLEDGMENT}

The authors wish to acknowledge the assistance and support of R. Clark, University of Surrey, Surrey, U.K., and the Processing Group, University of Oulu. Oulu, Finland, for fabricating the test antennas.

\section{REFERENCES}

[1] K. M. Lum, C. Laohapensaeng, and C. E. Free, "A novel traveling-wave feed technique for circularly polarized planar antennas," IEEE Microw. Wireless Compon. Lett., vol. 15, no. 3, pp. 180-182, Mar. 2005.

[2] K. M. Lum, T. Tick, C. Free, and H. Jantunen, "Design and measurement data for a microwave $\mathrm{CP}$ antenna using a new traveling-wave feed concept," in Eur. Microw. Conf., Paris, France, Oct. 2005, pp. 261-264.

[3] J. Huang, "A technique for an array to generate circular polarization with linearly polarized elements," IEEE Trans. Antennas Propag., vol. AP-34, no. 9, pp. 1113-1124, Sep. 1986.

[4] K. P. Tang, C. E. Free, and G. F. Goldspink, "A novel slotline-fed microstrip patch antenna," in IEEE Antennas Propag. Conf., Apr. 1995, vol. 1 , no. 407 , pp. 235-238.

[5] E. A. Mariani, C. P. Heinzman, J. P. Agrios, and S. B. Cohn, "Slot line characteristic," IEEE Trans. Microw. Theory Tech., vol. MTT-17, no. 12, pp. 1091-1096, Dec. 1969.

[6] J. B. Knorr, "Slot-line transitions," IEEE Trans. Microw. Theory Tech., vol. MTT-22, no. 5, pp. 548-554, May 1974.
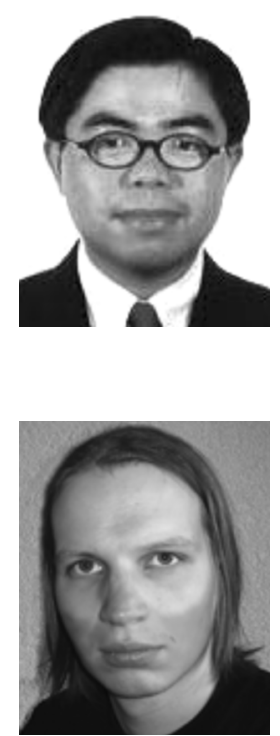

components.

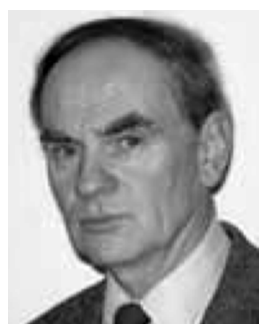

Charles Free (M'99) received the degree in electronic engineering.

He was a Research Engineer in industry and then moved to academia. He is currently a Lecturer of microwave technology with the University of Surrey, Surrey, U.K. He has authored or coauthored over 90 papers. His main research interests are in the design and characterization of planar microwave circuits.

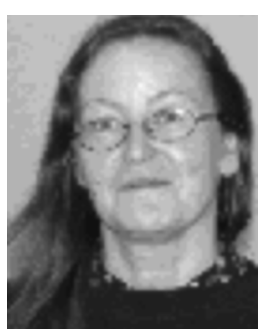

Heli Jantunen was born in Oulu, Finland, in 1958. She received the M.Sc. degree in astronomy and physics, Diploma in education, M.Sc. degree in electrical engineering, and Dr. Tech. degree in electrical engineering (with honors) from the University of Oulu, Oulu, Finland, in 1982, 1985, 1989, and 2001, respectively.

She is currently a Professor with the Microelectronics and Materials Physics Laboratories, University of Oulu. Since 2004, she has been the Group Leader of the Electronics Materials, Packaging and Reliability Techniques (EMPART) Research Group, Infotech Oulu, Oulu, Finland. Her research is focused on the design, development, synthesis, and implementation of electronics materials and their components for RF and microwave applications, as well as multifunctional micromodules. 